\title{
Interdisziplinäre Fortbildung Kinderdermatologie 2006 - Neurodermitis und Akne, 15. November 2006, Orangerie Aukamm Wiesbaden
}

\author{
Interdisciplinary Symposium Pediatric Dermatology 2006 - Atopic Dermatitis and Acne, 15. November 2006, \\ Orangerie Aukamm Wiesbaden
}

\section{Autor}

Institut

\section{Bayerl}

Klinik für Dermatologie und Allergologie, HSK Wilhelm-Fresenius-Klinik, Städtisches Klinikum Wiesbaden

\section{Bibliografie}

Dol $10.1055 / \mathrm{s}-2007-966380$

Akt Dermatol 2007; 33:

123-125 @ Georg Thieme

Verlag KG Stuttgart · New York ISSN 0340-2541

Korrespondenzadresse

Prof. Dr. med. Christiane Bayerl

Klinik für Dermatologie und Allergologie

Städtische Kliniken Wiesbaden

Lehrkrankenhaus der

Universität Mainz

HSK, Wilhelm-Fresenius-Klinik $\mathrm{GmbH}$

Aukammallee 39

65191 Wiesbaden christiane.bayerl@hsk-

wiesbaden.de
Die Klinik für Dermatologie und Allergologie (Direktorin Prof. Dr. med. Christiane Bayerl) gemeinsam mit der Klinik für Kinder und Jugendliche (Direktor Prof. Dr. med. Michael Albani) der Dr. Horst-Schmidt Kliniken des Städtischen Hauses der Stadt Wiesbaden verfolgten mit ihrem Fortbildungsangebot für zuweisende Kollegen neue Wege. Zusammen organisierten sie eine Fortbildung zu wichtigen Fragestellungen des Kindesund Jugendalters, zu Neurodermitis und zu Akne. Die Veranstaltung war integriert in die Reihe „Wiesbadener Dermatologenabende“, aber diesmal interdisziplinär für Dermatologen und Kinderärzte und zertifiziert für beide Fachrichtungen. Die erfreulich hohe Besucherzahl von 200 Teilnehmern bestätigte die Organisatoren in dieser Themenwahl.

Im ersten Referat gab Frau Prof. Dr. med. Christiane Bayerl, Direktorin der Klinik für Dermatologie und Allergologie Wiesbaden, einen Überblick über neue epidemiologische Daten zu Allergien im Kindesalter. Mittlerweile leidet jedes vierte Kind an einer Erkrankung des atopischen Formenkreises. In einem Beobachtungszeitraum von einem Jahr zeigten epidemiologische Studien bei 13-14-jährigen Kindern das Auftreten von Symptomen für die allergische Rhinokonjunktivitis bei $13 \%$, für das allergisches Asthma bronchiale bei $15 \%$ und für die Neurodermitis bei $7 \%$. Im internationalen Vergleich liegt Deutschland damit an mittlerer Stelle. Zwillingsstudien haben gezeigt, dass erbliche Faktoren bei der Neurodermitis eine Rolle spielen, das Krankheitsbild in Ausprägung, Verlauf und Schweregrad jedoch von Umweltfaktoren bestimmt wird. Diese können Allergene aus der Umwelt sein, Hausstaubmilben, Schimmelpilze, Haustiere, Irritantien wie Wolle oder Feuchtarbeit, Infektionen, Stress, aber auch „Langeweile“. Zwar verlieren ca. 60\% der Kinder bis zur Pubertät ihre Neurodermitis, jedoch entwickeln 30\% der Kinder mit Neurodermitis während der Pubertät ein allergisches
Asthma bronchiale. Eine schwedische Studie (Bradley M et al., Acta Derm Venereol 2000, $80: 106$ - 110) zeigte, dass bei fast $70 \%$ der Zwillinge mit Neurodermitis eines der Elternteile oder beide ebenfalls eine Neurodermitis hatten. Bei Neurodermitis-Kindern mit nur einem erkrankten Elternteil hatte bei $37 \%$ der Vater die Neurodermitis, bei $63 \%$ die Mutter. Mütter mit Neurodermitis vererben die Erkrankung also doppelt so häufig wie Väter mit Neurodermitis. Dazu gibt es einige Hypothesen: die genetische Determinierung, Umweltfaktoren in utero oder während der Stillzeit sowie eine höhere Schwelle bei Männern, auf Umweltreize hin das Krankheitsbild zu entwickeln. So sind Eltern verständlicherweise interessiert, bereits im Säuglingsalter Maßnahmen zu ergreifen, die das Risiko ihres Kindes, eine Neurodermitis zu entwickeln, minimieren oder schwere Verlaufsformen verhindern helfen. Immer noch gilt die Empfehlung, Hausstaubmilben zu sanieren und Schimmel und Feuchtigkeit in der Wohnung zu bekämpfen. Aktuelle Studien belegen, dass Rauchen der Mutter während der Schwangerschaft und danach und die Passivrauchexposition das Allergierisiko für Kinder erhöhen. Dieselruß aus Straßenverkehrsemissionen und das Katzenallergen sind weiterhin zu meiden. Das Halten vieler Hunde, das Großwerden auf dem Bauernhof und der Besuch der Kindertagesstätte wirken nur in den ersten Lebensmonaten protektiv, im Kindergartenalter haben diese Maßnahmen keinen schützenden Effekt mehr. Dahinter steckt die Theorie, dass sich eine frühe unspezifische Immunmodulation günstig auf die immunologische Entwicklung eines Kindes auswirkt. Kein Zusammenhang besteht zwischen Antibiotikaeinnahme und Allergieentstehung. Unbedingt eingehalten werden sollten die aktuellen Impfempfehlungen - auch bei Allergikern!

Herr Dr. med. Ulrik Winckelmann, OA der Klinik für Kinder und Jugendliche Wiesbaden, sprach 
zur Frage der hypoallergenen Ernährung im Säuglingsalter (siehe Seite 126). Immer noch lautet die Empfehlung für Allergikerfamilien, in den ersten sechs Lebensmonaten zu stillen. Ist dies nicht möglich, bieten aber auch Proteinhydrolysate, teils oder extensiv hydrolysierte Zubereitungen oder solche basierend auf Molke oder Casein und die strikte Meidung von Kuhmilch einen Schutz vor Atopieentwicklung. Bei nachgewiesener Milchallergie und nur dann werden auf Aminosäuren aufgebaute oder so genannte Elementardiäten empfohlen. Die Zugabe von Beikost und fester Nahrung sollte nach und nach erfolgen und am besten erst nach dem sechsten Lebensmonat. Sinn macht die Karenz hochallergener Nahrungmittel wie Ei, Milch, Soja, Fisch und Nüsse im ersten Lebensjahr bei Risikokindern. Dagegen besteht keine Notwendigkeit, dass die Mutter während der Schwangerschaft hochallergene Nahrungsmittel meidet. Erste Studien empfehlen bei Kindern mit Atopierisiko probiotische Ernährung in der Schwangerschaft und für stillende Mütter und präbiotische Ernährung für Kinder, die nicht gestillt werden.

Herr Prof. Dr. med. Dietrich Abeck, Dermatologe in München, löste mit seinem Referat zu den Essentials im Management der Neurodermitis eine nicht enden wollende Flut an Fragen aus. Eine Abheilung der Ekzeme ist häufig auch unter einer konsequenten Basistherapie nicht möglich, so dass zusätzlich antientzündlich wirksame Substanzen zum Einsatz gelangen müssen (siehe Seite 135). Die antiinflammatorische Behandlung mit Steroiden und topischen Calcineurin-Inhibitoren ist ein wichtiger Baustein der Lokaltherapie. Leider stehen weitere vergleichbar wirksame Alternativen nicht zur Verfügung. Dies gilt für Gerbstoff-, Phytopharmaka- oder für schieferölhaltige Externa. Neben einer geringen Wirksamkeit besteht für Bufexamac zusätzlich das Risiko einer allergischen Reaktion. Eine wichtige Empfehlung ist, die Hautflora seitens des Befalls mit dem grampositiven Bakterium Staphylococcus aureus zu sanieren. Dies gelingt durch den Einsatz von Antiseptika wie z.B. Triclosan und/oder Chlorhexidin. Neben dem Einsatz von Antiseptika wirken sich funktionelle Textilien mit antimikrobiellen Zusätzen in Form von mit einer quarternären Ammoniumverbindung beschichteten Seide oder einer silberbeschichteten Mikrofaser gut auf die Keimbesiedlung der Haut aus.

Frau PD Dr. med. Sonja Ständer, OÄ der Hautklinik Münster, hatte im Zentrum ihres Vortrags den quälenden Juckreiz bei Neurodermitis (siehe Seite 131). Bei Atopikern ist die Juckreizschwelle erniedrigt. Ursache dafür ist die erhöhte Sensibilität der schnell leitenden A-Fasern und der langsam leitenden C-Fasern der Haut. Durch diese ständige Aktivierung der Juckreizbahnen kommt es zur neuen Aufgabedefinition der ganz in der Nähe liegenden schmerzleitenden Fasern, die dann plötzlich Juckreiz leiten und damit die Chronifizierung fördern. Dann helfen nur noch zentral wirksame Substanzen, was sich wiederum im Kindesalter verbietet. Das Symptom Juckreiz wird in seiner pathophysiologischen Auslösung durch neurohormonale Faktoren in der Haut mittlerweile besser verstanden und bietet daher therapeutische Angriffspunkte. Histamin und Acetylcholin provozieren Juckreiz über eine direkte Bindung an bestimmte Juckreizrezeptoren. Verschiedene Mediatoren wie Neuropeptide, Proteasen und Zytokine provozieren Juckreiz indirekt über Freisetzung von Histamin. Die Haut atopischer Kinder enthält vermehrt Aund C-Fasern, aber darüber hinaus auch vermehrt Neuropeptide und Neuropeptidrezeptoren. Als Vermittler des Juckreizes werden bei Neurodermitis nicht mehr vorrangig das Histamin, sondern vor allem Tryptase und PAR-2 angesehen. Therapeutische Ansatzpunkte liegen im Cannabinoid-System der Haut. Capsai-

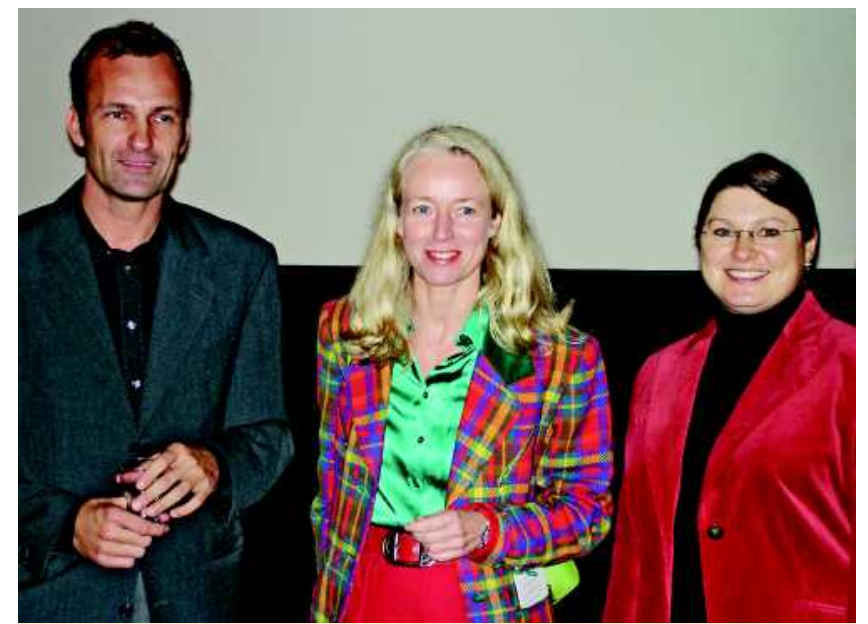

Abb. 1 Die Referenten/innen des ersten Veranstaltungsteils zum Thema Neurodermitis, Herr Dr. med. Ulrik Winckelmann, OA der Klinik für Kinder und Jungendliche Wiesbaden, Frau Dr. med. Christiane Bayerl, Direktorin der Klinik für Dermatologie und Allergologie Wiesbaden und Organisatorin der Veranstaltung und Frau PD Dr. med. Sonja Ständer, OÄ der Hautklinik Münster.

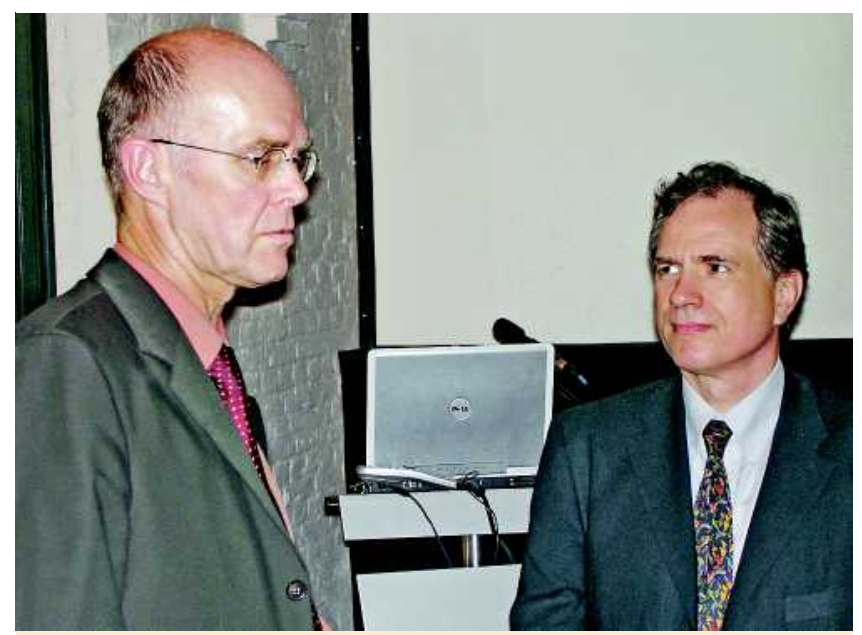

Abb. 2 Die Referenten der zweiten Hälfte der Veranstaltung zum Thema Akne, Herr Prof. Dr. med. Uwe Gieler, Zentrum für Psychosomatische Medizin Gießen und Herr Prof. Dr. med. Uwe Ochsendorf, Ltd. OA der Hautklinik Frankfurt.

cin ist eine Therapieoption für Jugendliche. Für alle Alterklassen verfügbar ist ein topischer Cannabinoidagonist mit Palmitaminmonoethanolamin (PEA), der bei Pruritus und Prurigo nodularis in Anwendungsbeobachtungen erfolgreich war.

Im zweiten Teil der Veranstaltung ging es dann um die „Pickel“ in der Pubertät. Herr Prof. Dr. med. Falk Ochsendorf, Ltd. OA der Hautklinik Frankfurt, berichtete über aktuelle Aspekte in Pathogenese und Therapie der Acne vulgaris (Seite 137). Die Prävalenz bei Jugendlichen liegt bei bis zu $80 \%$. Die Akne ist keine kosmetische Störung, sondern eine Erkrankung der Haut, bei der genetische Faktoren eine große Rolle spielen. Das moderne pathogenetische Konzept versteht die Akne nicht mehr als vorrangig bakteriell bedingt, sondern vor allem als eine immunologische Reaktion. Die Ursache liegt in Seborrhoe, Verhornungsstörung, mikrobieller Besiedelung mit Propionibacterium acnes und der Entzündungsreaktion. Eine effektive Behandlung orientiert sich am klinischen Bild. So werden Verhornungsstörungen mit topischen Retinoiden und Benzoylperoxid (BPO), bakterielle Besied- 
lung mit topischen und systemischen Antibiotika und BPO und Entzündungen mit Antibiotika behandelt. Mit Antiandrogenen erreicht man die Seborrhoe. Mit Isotretinoin werden alle pathogenetischen Faktoren erfolgreich angegangen. Kombinationsbehandlungen mit verschiedenen der genannten Substanzen haben sich bewährt.

In der Pubertät ist nicht nur die Haut, sondern auch die Psyche reaktiv „mitgenommen“. Entsprechend behandelte das Symposium nicht nur die moderne topische und medikamentöse Therapie der Akne, sondern auch psychosomatische Problemfelder wie z.B. „Akne, Pickel, doofe Eltern“, so der Titel des Referats von Herrn Prof. Dr. med. Uwe Gieler, Zentrum für Psychosomatische Medizin Gießen. Akne kann Depressionen und soziale
Ängste auslösen. Compliance in der Behandlung zu erreichen, ist die größte Herausforderung für den Dermatologen, der Pubertierende behandelt. Bekannt ist, dass nur 70\% der jungen Aknepatienten die Therapie durchhalten. Nur ein Behandlungskonzept, das im Gespräch erarbeitet wurde und auch vom Patienten akzeptiert wird, führt zum Erfolg. Bewährt hat sich, den Patienten am Ende eines Gesprächs nochmals wiederholen zu lassen, was vereinbart wurde.

Diese erste interdisziplinäre Wiesbadener Fortbildung Kinderdermatologie 2006 ist einzigartig in ihrem Konzept, in Wiesbaden initiiert, und wird aufgrund des großen Erfolges 2008 erneut stattfinden.

\title{
Buchbesprechung
}

\author{
Allergiediagnose, Allergietherapie \\ Trautmann A \\ Stuttgart: Thieme, 2006. 358 S., 75 Abb. Kart. 39,95€, ISBN 3-13-142181-9
}

„Allergiediagnose, Allergietherapie“ - der Allergiebegriff ist als schillerndes Modewort in aller Munde und wird oft genug überstrapaziert. Dem wirkt das Paperback entgegen durch klare, übersichtliche Definitionen der Grundbegriffe und notwendige Abgrenzungen. Dazu zählen Unverträglichkeitsreaktionen wie Pseudoallergien, Intoleranzen, Idiosynkrasie bis zu psychogenen Reaktionen. Sie verwischen die Grenzen des Allergiebegriffs durch eigene, nicht-allergische Mechanismen, die aber große Ähnlichkeit der klinisch sichtbaren Bilder aufweisen.

Grenzüberschreitend sind auch die fachlichen Zuständigkeiten für die Allergiepatienten. Dermatologische, $\mathrm{HNO}$-ärztliche und pulmologische sowie pädiatrische Aspekte finden Eingang in das übersichtliche Checklistenformat mit Kapiteln wie Ekzeme, Rhinitis allergica, allergisches Asthma bronchiale. Nach einheitlichem Schema werden übersichtlich Anamnese, klinische Befunde, Labor- und klinische Tests bis zur Therapie dargestellt. 75 Abbildungen und Schemata, z. B. zu Allergengruppen und Kreuzallergien (auch weniger bekannte wie Vogel-Ei und Katze-Schwein-Kreuzallergie) und zu Hyposensibilisierungsplänen sowie Tabellen lockern den vielfach stichwortartigen verdichteten Text auf und vermitteln auf einen Blick entscheidende Informationen. Beispielsweise kann die Auflistung von Medikamenten, unter denen z.B. die Prick-Testung kontraindiziert oder in ihrer Aussagekraft reduziert ist, in ihrem Wert nur schätzen, wer sich derlei Detailwissen für die tägliche praktische Anwendung bisher aus Einzelpublikationen zusam- mensuchen musste. Ebenso die Schemata zu den aktuellsten Hyposensibilisierungsempfehlungen: solche „Spickzettel“ entspringen umfassenden Erfahrungen des Autors aus der klinisch-allergologischen Ambulanztätigkeit und treffen exakt ärztliche Bedürfnisse im täglichen Praxisbetrieb.

Die Angaben zur Therapie bzw. Prophylaxe (oder auch die Sammlung von Internetadressen) dokumentieren die Aktualität des Buches, z.B. auch durch die Aufführung der Hyposensibilisierungspräparate auf dem neuesten Stand. Angesichts der rasanten Entwicklung neuer Erkenntnisse und Empfehlungen gerade in diesem Bereich - etwa bei der rush-Hyposensibilisierung ... - sind dies unverzichtbare Qualitäten des Buches zur Absicherung des Anwenders und seiner Patienten.

Für eine künftige Auflage wären die neuen Möglichkeiten oraler Hyposensibilisierung z. B. gegen Gräserpollen eine - sei es auch kritische - Würdigung wert. Darüber hinaus könnte eine Liste der gängigsten ECT-Testreihen die wertvollen tabellarischen Angaben des Buches abrunden.

Zusammenfassend hat man sich für die tägliche allergologische Praxis einen solchen Helfer und Ratgeber schon lange gewünscht. Sein moderater Preis kontrastiert mit dem hohen Praxiswert. Darüber hinaus bietet das Buch aber auch zahlreiche prüfungsrelevante Fakten. Damit wird es auch als Ergänzung zu Lehrbüchern bzw. als Repetitorium vor der Prüfung für die Zusatzbezeichnung Allergologie dankbar angenommen werden.

Bettina und Dieter Krahl, Heidelberg 